\title{
Researching Continuity of Care: Can Quality of Life Outcomes be Linked to Nursing Care?
}

\section{Authors:}

Professor Anne McMurray, R.N., Phd, FRCNA - Professor of Nursing, Research Centre for Clinical Practice Innovation, Griffith University, PMB 50 Gold Coast Mail Centre, Southport, Queensland, 9726.

A.McMurray@griffith.edu.au Phone (07) 55766941, mobile 0409587850.

Dr. Karen Theobald, R.N., Phd., MHSc (Nurs), G.Cert (Higher Ed), B.App. Sc.

Lecturer, Queensland University of Technology,

Kelvin Grove Campus, Brisbane, 4001.

K.Theobald@qut.edu.au, Phone (07) 39015956.

Associate Professor Wendy Chaboyer, R.N., Phd, FRCNA, Director, Research Centre for Clinical Practice Innovation, Griffith University, PMB 50 Gold Coast Mail Centre, Southport, Queensland.

W.Chaboyer@griffith.edu.au Phone (07) 55528518. 


\title{
Researching Continuity of Care: Can Quality of Life Outcomes be Linked to Nursing Care?
}

\begin{abstract}
Research that informs nursing interventions across the care continuum is vital, especially with shortened hospital stays. Measuring Quality of Life (QOL) and Health Related Quality of Life (HRQOL) helps identify health status improvements, but fails to provide insight into the effectiveness of nursing interventions aimed at continuity of care. Four research examples illustrate the need for complementary, qualitative studies of what patients and their families think, feel, need and want. These indicate a need to reconceptualise the research agenda in terms of the complexity and settings of nursing practice, and the need for informational as well as statistical significance.
\end{abstract}

Key words: Quality of life, Health-related quality of life, continuity of care, patient experiences, illness and recovery, home and community care, health transitions. 


\section{Researching Continuity of Care: Can Quality of Life Outcomes be Linked to Nursing Care?}

\section{Introduction}

This article examines the usefulness of measuring 'Quality of Life' (QOL) and 'Health-Related Quality of Life' (HRQOL) in researching continuity of patient care across the hospital to home and/or community continuum. Continuity of care refers to connected client-care events between settings (Sparbel \& Anderson, $2000 \mathrm{a}, \mathrm{b}$ ). It is a major goal of nursing practice and the main objective of discharge planning protocols, especially with patients being released from hospital 'quicker and sicker'.

Research into continuity of care has been inconsistent, with some studies addressing only medical care, and others investigating access to care or services, patient and carer satisfaction with care, rates of hospital readmission, or models for coordinating treatment (Anderson, Hanson, DeVilder \& Helms, 1996; Canadian Health Services Research Foundation, 2001; Clare \& Hofmeyer, 1998; Heaton, Arksey \& Sloper, 1999; Montalto, 1996). The nursing literature reveals a growing number of studies, including our own, that have used QOL and HRQOL as measures of client self-assessed health during recovery from illness or surgical intervention (Anderson, Feleke \& Perski, 1999; Chaboyer \& Elliott, 2000; Jaarsma \& Kastermans, 1997; Knutsson \& Bergbom Engberg, 1999; McMurray, Grant, Letford \& Griffiths, 2002). These and other studies measuring health status during recovery (Mistiaen et al, 1997; Ridge \& Goodson, 2000) indicate a need for greater precision and clarity in the language used to conceptualise clients' health related progress over time and across settings. Below we describe four studies in our own programs of research that argue for further debate to inform conceptual development in this line of nursing research. 


\section{QOL and HRQOL Research}

The term QOL has come to refer to everything from the "relative liveability" of communities to states of health and health service provision (Rapley, 2003, p. xiii). In health research, QOL includes not only functional consequences of health, but subjective feelings about life satisfaction, happiness and the overall value one places on life at any given time (Cella \& Nowinski, 2002). Such ambiguity challenges QOL researchers to work toward conceptual clarity and refinement of measurement instruments. To date, 447 measures are listed by the Australian Centre on Quality of Life (Rapley, 2003). In some cases, health status measures have been used to measure QOL and HRQOL, but this makes it difficult to argue the findings, because perceptions of health status may be confounded by other factors, such as satisfaction with family circumstances, or with outcomes of care (Ager, 2002; Bradley, 2001; Bradley \& Tamburini, 2003; Dunn, Lynch, Aitken, Leggett, Pakenham \& Newman, 2003; Williams, Coyle \& Healy, 1998). Health status is therefore a narrow conceptualisation of what is encompassed in QOL. Some researchers argue that although QOL and HRQOL measures are important in gaining patients' perspectives of the outcomes of care, the focus of questions is actually on perceptions of health and wellbeing rather than the broader notion of QOL (Kahn \& Juster, 2002). Using a health status measure to link improvements in QOL to clinical interventions is particularly problematic because, although improvements in health status can be an indication of successful clinical judgements and interventions, they may also result from other influences beyond the control of nurses and other health professionals.

It is important to examine the relationship between health status and QOL in nursing studies to investigate whether improvements in self-perceived health status and/or QOL can be linked to the use of nursing services or the effectiveness of 
nursing interventions. Until these relationships are made explicit there will be a lack of conceptual clarity in investigating continuity of care. This area of research informs both institutional and home care, and it achieves a good 'fit' with the Evidence-Based Practice (EBP) and Evidence-Based Nursing (EBN) agendas, which recommend that both observed and research-derived evidence for practice should be moderated by knowledge of patient circumstances and preferences (Carnwell, 2000).

\section{Quality of Life: Conceptual Clarity}

QOL has been central to health care research throughout the latter part of the last century, and since the 1980's, studies in this area have proliferated (Aaronson et al., 1991; Schipper, Clinch \& Olweny, 1996; Garratt, Schmidt, Mackintosh \& Fitzpatrick, 2002). Oncology research provided an impetus for QOL studies, in recognition that optimal medical care must include QOL considerations (Aaronson et al., 1991). This view is aligned with the ethos of palliative care, disability studies and chronic illness management, and it has spawned research in other medical and health specialties (Cella \& Nowinski, 2002; Kaasa \& Havard Loge, 2003; Karlawish, Casarett, Klocinski \& Clark, 2001; Lefort \& Fraser, 2002; Sneeuw, Sprangers \& Aaronson, 2002; Speight, 2002; Tengs \& Lin, 2003).

QOL researchers agree that it is a multi-dimensional concept including functional status, psychological and social well being, health perceptions, and treatment related outcomes such as symptom relief (Aaronson et al., 1991; Cella \& Nowinski, 2002; Chaboyer \& Elliott, 2000; Kaasa \& Loge, 2003; Schipper et al, 1996). HRQOL instruments focus on the health aspects of QOL, focusing on either generic normative data or condition-specific benchmarks. Both are thought to be important in studying chronic illness management to maximise their complementarity (Cella \& Nowinsky, 2002). For example, the Western Ontario and McMaster 
Universities Osteoarthritis Index (WOMAC) is specific to arthritis and can discriminate levels of disability, while the Medical Outcomes Study Short Form 36 (SF-36) has a greater ability to discriminate different levels of general health status and the presence of co-morbidities (Cella \& Nowinsky, 2002).

Measures of QOL, HRQOL and health status have been used to investigate client recovery following an acute illness episode or treatment for a chronic condition because they are valid, sensitive to changes and represent the client perspective. The SF-36 (Ware \& Sherbourne, 1992), the Sickness Impact Profile (SIP) (Bergner, Bobbitt, Carter \& Gilson, 1981); the Nottingham Health Profile (NHP) (Hunt, McEwan \& McKenna, 1985), and the EuroQol, or EQ5D (EuroQol Group, 1990) are among the most widely used measures. A review by Garratt et al (2002) revealed 3921 reports describing client-assessed measures of QOL, but little standardisation among them. The SF-36, described by its authors as a health survey (Ware \& Sherbourne, 1992), is the most widely used (Garratt et al., 2002).

Problems with studying QOL revolve around concerns that the measures used are, in fact, indicators of how people feel about their health, rather than QOL (Bradley, 2001; Garratt et al, 2002; Lefort \& Fraser, 2002; Speight, 2002). Other difficulties lie in researching such a broad area. Ager (2002) argues that comprehensive measures tend to homogenise approaches to data collection, obscuring analysis of the core outcomes of clinical interventions. He and others contend that the breadth of coverage of QOL measures and the absence of a single, widely accepted definition of QOL may limit its usefulness by threatening statistical analysis and clinical interpretation of data (Dennerstein \& Helmes, 2000; Dunn et al., 2003; Kaasa \& Loge, 2003). This creates ambiguity and tension, especially when QOL measures are used in many different ways, including measures of both need and outcome 
(Dennerstein \& Helmes, 2000; Wilkin, Hallan and Doggett, 1992). For example, a low score on the physical or somatic component of QOL scales may indicate either the need for pain relief, or the outcome of having administered analgesics (Wilkin, Hallan and Doggett, 1992). Another issue relates to measuring patient satisfaction. Instead of reducing satisfaction to a minimum standard, salient features of context, process and experience should also be included, so that there is clarity in whether satisfaction refers to respondents' own progress, access to appropriate care or the conduct of the health service provider (Wilkin, Hallan and Doggett, 1992).

Kahn \& Juster (2002:628) equate QOL with wellbeing, but caution that both terms are "elusive concepts". Conceptual difficulties arise from the fact that wellbeing and happiness are relatively stable even during objective changes, such as occurs in illness. Kahn \& Juster (2002) identify five sources of potential bias in self-reported assessments of either wellbeing or QOL. First, assimilation can occur, where individuals recall a happy incident that heightens feelings of life satisfaction. Alternatively, the opposite occurs. A third source of bias may flow from a transient mood evoked by the questions. Fourth, responses may indicate social acceptability bias, where silent comparisons with others (e.g. in their age group) are made, or respondents make comparisons of themselves at another age or in another state of health. To enhance accuracy, both objective and subjective definitions of of the construct being measured must be made explicit.

Ruta, Garratt and Russell (1999) add another insight, conceptualising QOL as the difference between expectations and reality. Their view of QOL is the extent to which goals and expectations give meaning and purpose to life, and health care should narrow the gap between the two (Calman in Ruta et al., 1999). Lefort and Fraser (2002) describe QOL as an organising concept in public policy and service delivery, 
but they caution against the value-laden nature of the term QOL, given that it may lead to the notion that some people are more valuable than others. Such a notion could be used as a warrant for selectivity in the abortion of particular foetuses, the use of life-sustaining measures or physician-assisted suicide (Rapley, 2003). The World Health Organization (WHO, 2002) concurs, urging people's involvement in decisions about health and service delivery, and they suggest that researchers should ask what clients think, feel, want and need. This resonates with that aspect of the nursing agenda aimed at evaluating the outcomes of nursing care across the care continuum.

\section{Implications for Nursing Research}

With shortened hospital stays, the need to plan and 'track' client needs across the care continuum are major nursing concerns (Clare \& Hofmeyer, 1998). For nurse researchers, the mandate is to unravel the complexities of coordinating care, to investigate client needs and preferences for service delivery, their satisfaction with care, and ultimately, to build a body of evidence for good and best practice in assisting clients through their health and illness transitions. This suggests a research agenda wherein clients are asked directly what they think, feel, want and need at multiple points in time. Their responses could bring the agenda back to basics to provide better linkages between nursing practices and client outcomes. Corbin (2003) concurs, arguing that the meanings individuals assign to being ill or injured lead to vastly different insights into healthcare than those arising from the measurement of a quantitative variable.

Investigating the effectiveness of nursing interventions across the care continuum presents a special set of research challenges. One of these is the difficulty associated with following clients across multiple settings, especially with shortened hospital stays and streamlined management practices that facilitate client throughput 
by using separate pre-admission and pre-discharge personnel. These practices increase the efficiency of hospital processes, but leave little time to establish nurse researcherclient relationships or recruitment strategies. Another difficulty is the complex nature of nursing practice, where even brief nursing interventions attempt to identify client needs holistically, encompassing social and cultural, physical and psychological care. Without using global measures of health outcomes that are valid, sensitive to change and quickly administered, it is almost impossible to link acute or intermediate care with clinical outcomes. This calls into question the strength and meaningfulness of evidence that flows from data that cannot be benchmarked to standard measurements of self-perceived health status, such as the SF-36. Research studies that ask what people think, feel, want and need, have the capacity to generate important data to inform nursing practice; but the findings are of informational, rather than statistical significance. Given that the type of research favoured by most funding bodies is that which produces statistical significance, it is important to unravel the opportunity cost of favouring one or another of these approaches.

The studies below were chosen from our research programs to illustrate the need for a common research discourse. This would help facilitate development of a reconceptualized, cohesive body of knowledge informed by the realities of people's experiences and preferences. We argue that our research should be framed in terms of what people think, feel, want and need, rather than only in terms of QOL or HRQOL.

\section{Study Area 1: Family Health in Belmont.}

This study provided a case study 'snapshot' of one Western Australian community in 1997 which emerged from discussions among an interdisciplinary group of health professionals, each of whom had sought to identify locally defined health issues and patterns of health service need and use. Collectively we wondered 
what types of families preferred various services? Were most families accessing services that were appropriate for their needs? Did they feel they had a choice of services and what was the basis for their choosing one service over another? Was there a typical pattern of service use for families at various developmental stages? How well did people understand the potential of their health resources?

The findings of the study are reported elsewhere (McMurray, Hudson-Rodd, Al Khudairi \& Roydhouse, 1998), but their relevance to this discussion lies in the pathway to gaining the desired information. Despite considerable community planning, granting bodies declined to fund the study. The main criticism revolved around the lack of a valid data collection instrument, even though the proposed interview questionnaire had gone through several iterations and a pilot test for content validity. Although discouraged, we were not detracted. The participants readily responded, and were better informed about health services as a result. The findings were sent to various stakeholders, including the local health policy unit.

Among the useful (although not statistically significant) findings of this study was the preference for sole parent household families to take their sick children to the children's hospital rather than the child health nurse or the GP, because it was quicker and more accessible for them to have their children seen on the way to work. Another finding was that most people saw their family as relatively healthy, even when it was a caregiving family. In this type of family, both male and female carers found satisfaction in assisting their partners, which challenged our preconceived notion about the 'burden of care'. Other meaningful findings included the need for more and better health information from all health professionals.

\section{Study Area 2: Successful Recovery after CABG Surgery}


The objective of this study was to examine the range of post-discharge issues, concerns and needs of patients and their family carers following Coronary Artery Bypass Graft (CABG) surgery, and to explore their perceptions of unmet needs one year later. In other words, we were trying to determine what people think, feel, want and need at different stages across the recovery period. This information was intended to inform discharge planning processes that would promote continuity of care. The rationale for the study revolved around the fact that the rate of CABG surgery is steadily increasing and potentially life saving, with cardiovascular disease remaining the leading cause of death in Australia (AIHW, 2001). Again, no validated instrument was available to gather this information, so it was designed as a naturalistic, explorative study of two years duration.

Previous research in this area of cardiac surgery revealed common patient experiences, including a range of physical and psychosocial sequelae (Deaton, Weintraub, Ramsay, Przykucki, Zellinger \& Causey, 1998; Moore 1994, 1996). Studies also revealed a constellation of problems arising from family caregiving, often persisting for as much as one year following the cardiac event (Artinian, 1991, 1992, 1993; Monahan, Kohman \& Coleman, 1996; Moore, 1994). Most of the problems identified in previous research were also reported in our study, but the most remarkable finding was the need for better pre-operative preparation for both patients and carers. Study participants knew they were facing profound changes, but were unprepared for the actual experience of surgery, and for dealing with ongoing, unanticipated symptoms such as pain. Most were also unaware of the financial hardships they were to face in obtaining the services they required.

Like the Belmont Study (above) family members felt that what they wanted most from health professionals was accurate and appropriate information to help them 
through their health transitions and some way of connecting with others in similar circumstances (Theobald \& McMurray, 2003). The findings inform discharge planning, especially in preparing nurses to provide accurate guidance to families on how to navigate through the health care system following discharge from hospital.

\section{Study Area 3: HRQOL and Health Service Use Following Total Hip Replacement (THR) Surgery.}

This study was devised for similar purposes as the studies above: to inform nursing planning and clinical interventions across the care continuum. THR patients were the focus of this study, because, like cardiac patients, they represent a group requiring immediate and ongoing post-discharge care and follow-up. THR surgery has also been described as the most frequently occurring and successful type of surgery in terms of quality of life improvements, particularly for older persons who suffer from osteoarthritis (March, Cross, Lapsley, Brnabic, Tribe et al, 1999). The study was framed in QOL terms to investigate this claim, and to see whether any HRQOL improvements could be linked to the use of nursing and other health services. The method was also influenced by a previous application for funding, which had been rejected on the basis that it did not use validated instruments, with a recommendation that further applications consider using the SF-36.

The research team conformed to this mandate, given our commitment to identifying the information we sought and, rather than re-submit the proposal for national funding, we successfully applied for funding support from the State (Queensland) Nursing Council (QNC). The study findings revealed dramatic, early improvements in HRQOL scores, especially for mental health component scores, as

early as week 1 after discharge home. The findings did not allow us to link health improvements to health service use, but feedback from participants indicated that 
having ward nurses follow the patients through the post-discharge period for research purposes allowed ongoing communication with nurses, which enhanced continuity of care (McMurray et al, 2002).

In our view, the findings of this study will inform both practice and policy, particularly in planning health services. The information can be used as a basis for recommending increased health expenditure on information systems to track postsurgical patients across the health care system. With the current national research agenda targeting policy-ready research, the findings can contribute to EBP, and a follow-up study is currently in progress. Like the study of CABG patients and their carers, the THR study provides insight into the post-discharge home and community care experience and illustrates patient needs following an acute care episode.

\section{Study Area 4: Improving the Transitions for Critically Ill Patients}

We report here on a series of QOL studies conducted on the transition of patients from the Intensive Care Unit (ICU) to home (Chaboyer \& Grace, 2003; Chaboyer, Foster \& Creamer, 2002), and the impact of functional limitations on families (Foster \& Chaboyer, 2003). The importance of these studies lies in identifying, through SF-36 measures, that some ICU patients did not recover to their pre-illness health status (Chaboyer, Foster \& Creamer, 2002). Additional data revealed that carers provided an average of 38 hours per week in the first three months post hospital discharge where patients had prolonged ICU stays (Foster \& Chaboyer, 2003). Qualitative data obtained from telephone interviews and groups suggest that for some ICU survivors, poor sleep, pain, fatigue, weight loss and financial and social difficulties may last for years (Chaboyer \& Grace, 2003). While the SF-36 data identified some of the limitations in health status, the richness of the 
data gleaned from the qualitative research was more powerful in terms of understanding what these survivors thought, felt, wanted and needed.

This outcome of this research is development of a new nursing role, that of an ICU liaison nurse, to assist patients, families and nurses with the transition of patients from the ICU to the ward and home. The new role focuses on providing both practical support for patients and their families, and on educating and supporting nurses (Chaboyer, Foster, Kendall \& Foster, under review). Two aspects of the role include providing emotional support to anxious patients and identifying those patients who will most likely need follow-up assistance (Chaboyer, Foster, Kendall \& James, 2002, 2003). Difficulties encountered in this research have been in relation to pinpointing the actual patient outcomes that may be improved by this intervention. In fact, transfer anxiety, the main outcome variable, was unaffected by the new nursing role. At three months post-discharge, there was no difference between the intervention and control groups' SF-36 results, despite the fact that those who received the ICU liaison nursing service expressed tremendous satisfaction with the experience. This highlights the inherent difficulty in using QOL measures to demonstrate the effectiveness of nursing care. QOL is affected by so many factors, that the impact of any one factor, such as a nursing intervention, is difficult to measure. By simply measuring health status as opposed to asking patients what they think, feel, need and want, researchers are left to conclude that nursing interventions may be ineffective.

\section{Conclusion: Reconceptualising the Field of Study}

Valid QOL or HRQOL tools such as the SF-36 are likely to continue being used widely in medicine to chart clinical outcomes of interventions and inform good, best and cost effective medical practice (Krishnan \& Chipchase, 1997; McDaid, Cookson, Maynard \& Sassi, 2003). However, these measures do not demonstrate the total 
effects of either medical or nursing interventions, nor do they capture non-clinical influences on recovery, such as environmental and social circumstances. Their use in conjunction with other tools lies in cross-validating the concurrence of client and clinician judgements about health status, and this has proven helpful in the context of ensuring quality in electronic patient record systems (Bakken Henry, Lush, Constantino, Sousa, Mead \& Holzemer, 1997). Standardised measures of health status or HRQOL are also useful in benchmarking taxonomies of nursing interventions or classifications of nursing outcomes, despite lacking sensitivity at the extreme end of the scales, where the high acuity needs of older persons would be evident (Bakken Henry et al, 1997; Ridge \& Goodson, 2000).

Our review of QOL studies and our ongoing research into the link between QOL and continuity of care leads us to conclude that many studies designed to respond to the requirements of funding bodies fails to provide the depth of information needed to improve nursing practice. This does not diminish the importance of statistical significance measuring quantifiable variables of interest, but it argues for using complementary strategies to gain informational significance for nursing; that is, to be more nursing sensitive (Ridge \& Goodson, 2000). A second issue highlighted by this body of research is that the measurable impact of any one profession will always be difficult to demonstrate because of the number and complexity of factors influencing patient care. Equally as important, is the understanding that quantitative indicators do not always capture the importance individuals place on one or another measure. If researchers ask people to rate their health on a number of indicators, they will do so, but they may be using subtle weighting systems that are not revealed in our analyses. All of these issues point to the need to develop a broad conceptual framework within which to situate further studies in this line of research. This could best be achieved by 
encouraging ongoing dialogue among researchers interested in QOL and continuity of care to add greater depth and breadth to this important and expanding line of inquiry. 


\section{References}

Aaronson N, Meyerowitz B, Bard M, Bloom J, Fawzy F et al. (1991): Quality of life research in oncology. Cancer 67(3): 839-843.

Ager A (2002): 'Quality of life' assessment in critical context. Journal of Applied Research in Intellectual Disabilities 15: 369-376.

Anderson MA, Hanson K, DeVilder N \& Helms L (1996): Hospital readmissions during home care: a pilot study. Journal of Community Health Nursing 13 (1): $1-12$.

Anderson G, Feleke E \& Perski A (1999): Patient-perceived quality of life after coronary bypass surgery. Scandinavian Journal of Caring Sciences 13(1):1117.

Artinian N (1991): Stress experience of spouses of patients having coronary artery bypass during hospitalisation and 6 weeks after discharge. Heart and Lung 20(1): 52-59.

Artinian N (1992): Spouse adaptation to mate's CABG surgery: 1-year follow-up. American Journal of Critical Care 1(2): 36-42.

Artinian N (1993): Spouses' perceptions of readiness for discharge after cardiac surgery. Applied Nursing Research 6(2): 80-88.

Australian Institute of Health and Welfare (2001): Heart, stroke and vascular diseases, (AIHW cat.no.CVD 13): Australian Institute of Health and Welfare, National Heart Foundation of Australia and National Stroke Foundation of Australia, Canberra.

Bakken Henry S, Lush M, Constantino M, Sousa K, Mead C \& Holzemer W (1997): Health status measurement in computer-based patient record systems. Nursing Administration Quarterly 21(3): 50-60.

Bergner M, Bobbitt R, Carter W \& Gilson B (1981): The sickness impact profile: Development and final revision of a health status measure. Medical Care 19: 787-805.

Bradley C (2001): Importance of differentiating health status from quality of life. Commentary, The Lancet 357: Jan. 6, 7-8.

Bradley C \& Tamburini M (2003): Not only-a-title. Commentary, Health and Quality of Life Outcomes 1:1.

Canadian Health Services Research Foundation (2001): Summary of definitions of continuity of care. Accessed online July 12 2002, at http://www.chsrf.ca/docs/finalrpts/index_e.shtml. 
Carnwell R (2000): Essential differences between research and evidence-based practice. Nurse Researcher 8(2): 55-63.

Cella D\& Nowinski C (2002):Measuring quality of life in chronic illness: The functional assessment of chronic illness therapy measurement system, Archives of Physical Medical Rehabilitation 83 (Suppl 2): 10-S17.

Chaboyer W, Foster M, Creamer J (2002): Health status of ICU survivors: A pilot study. Australian Critical Care 15(1): 21-26.

Chaboyer W, Foster M, Kendall E \& Foster M: The Intensive Care Unit liaison nurse: Towards a clear role description (under review).

Chaboyer W, Foster M, Kendall E \& James H: The impact of a Liaison Nurse on ICU Nurses' perceptions of discharge planning (under review).

Chaboyer W, Foster M, Kendall E \& James H. (2002):ICU Nurses' Perceptions of Discharge Planning: A Preliminary Study. Intensive and Critical Care Nursing 18:90-95.

Chaboyer W, \& Grace J (2003): Following the pain of ICU survivors: A quality improvement activity. Nursing in Critical Care 8: 149-155.

Chaboyer W, Kendall E \& Foster M. (2002): Use of the Blaylock Risk Assessment Screening Score to Identify ICU Patients Who May Have Complex Hospital Discharge Planning Needs. Nursing in Critical Care 7:171-175.

Chaboyer W \& Elliott D (2000): Health-related quality of life of ICU survivors: review of the literature. Intensive and Critical Care Nursing 16: 88-97.

Clare J \& Hofmeyer A (1998): Discharge planning and continuity of care for aged people: Indicators of satisfaction and implications for practice. Australian Journal of Advanced Nursing 16(1): 7-13.

Corbin J (2003): The body in health and illness. Qualitative Health Research_13(2): $56-267$.

Deaton C, Weintraub W, Ramsay J, Przykucki R, Zellinger M \& Causey K (1998): Patient perceived health status, hospital length of stay, and readmission after coronary artery bypass surgery. Journal of Cardiovascular Nursing 12(4): 6271.

Dennerstein L \& Helmes E (2000): The menopausal transition and quality of life: Methodologic issues. Quality of Life Research 9 (suppl 1): 721-731.

Dunn J, Lynch B, Aitken J, Leggett B, Pakenham K \& Newman B (2003): Quality of life and colorectal cancer: A review. Australian and New Zealand Journal of Public Health 27(1): 41-46. 
EuroQol Group (1990): EuroQol: A new facility for the measurement of healthrelated quality of life. Health Policy 16: 208.

Foster M \& Chaboyer W (in press). Family carers of ICU survivors: A survey of the burden they experience. Scandinavian Journal of Caring Science.

Garratt A, Schmidt L, Mackintosh A \& Fitzpatrick R (2002): Quality of life measurement: Bibliographic study of patient assessed health outcome measures. BMJ 324 (7351): 1417-1424.

Harrison M, Browne G, Roberts J, Tugwell P, Gafni A \& Graham I (2002): Quality of life of individuals with heart failure: A randomised trial of the effectiveness of two models of hospital-to-home transition. Medical Care 40: 271-282.

Heaton J, Arksey H \& Sloper P (1999): Carers' experiences of hospital discharge and continuing care in the community. Health and Social Care in the Community 7(2): 91-99.

Hunt S, McEwen J \& McKenna S (1985): Measuring health status: a new tool for clinicians and epidemiologists. Journal of College of General Practice, 35: 15-188.

Jaarsma T \& Kastermans M (1997): Recovery and quality of life one year after coronary artery bypass grafting. Scandinavian Journal of Caring Science 11: 21-27.

Kaasa S \& Havard Loge J (2003): Quality of life in palliative care: principles and practice. Palliative Medicine 17 (1): 11-12.

Kahn R \& Juster F (2002): Well-being: concepts and measures. Journal of Social Issues 58 (4): 627-644.

Karlawish J, Casarett D, Klocinski J \& Clark C (2001): The relationship between caregivers' global ratings of Alzheimer's disease patients' quality of life, disease severity, and the caregiving experience. Journal of the American Geriatrics Society 49: 1066-1070.

Kilgore K (1995): Measuring outcomes in the postacute continuum. Archives of Physical Medical Rehabilitation 76: SC21-26.

Knutsson S \& Bergbom Engberg I(1999): An evaluation of patients' quality of life before, 6 weeks and 6 months after total hip replacement surgery, Journal of Advanced Nursing 30 (6): 1349-1359.

Krishnan J \& Chipchase L (1997): Orthopaedic surgery outcomes assessment model. Journal of Quality Clinical Practice 17: 109-116.

Lefort S \& Fraser M (2002): Quality of life measurement and its use in the field of learning disabilities. Journal of Learning Disabilities 6 (3): 22-38. 
March L, Cross M, Lapsley H, Brnabic A, Tribe K, Bachmeier C, Courtenay B \& Brooks P (1999): Outcomes after hip or knee replacement surgery for osteoarthritis. Medical Journal of Australia 171 (6 Sept): 235-238.

McDaid D, Cookson R, Maynard A \& Sassi F (2003): Evaluating health interventions in the $21^{\text {st }}$ century: Old and new challenges. Health Policy 63(2):117-120.

McMurray A., Hudson-Rodd N, Al Khudairi S \& Roydhouse R (1998): Family health and health services utilisation in Belmont, Western Australia: A community case study. Australian and New Zealand Journal of Public Health 22 (1): 107114.

McMurray A, Grant S, Letford A \& Griffiths S (2002): Health-related quality of life and health service use following total hip replacement surgery. Journal of Advanced Nursing 40 (6): 663-672.

Mistiaen P, Duijnhouwer E, Wijkel D, de Bont M \& Veeger A (1997): The problems of elderly people at home one week after discharge from an acute care setting. Journal of Advanced Nursing 25: 1233-1240.

Monahan D, Kohman L \& Coleman M (1996):Open-heart surgery: Cnsequences for caregivers. Journal of Gerontological Social Work 25 (3/4): 53-70.

Montalto M (1996): Patients' and carers' satisfaction with hospital-in-the-home care. International Journal for Quality in Health Care 8 (3): 243-251.

Moore S (1994): Psychologic distress of patients and their spouses after coronary artery bypass surgery. AACN Clinical Issues in Critical Care Nursing 5 (1): 59-65.

Moore S (1996): The effects of a discharge information intervention on recovery outcomes following coronary artery bypass surgery. International Journal of Nursing Studies 33 (2): 181-189.

Rapley M. (2003): Quality of Life Research: A Critical Introduction. Sage, London.

Ridge R \& Goodson A (2000): The relationship between multidisciplinary discharge outcomes and functional status after total hip replacement. Orthopaedic Nursing 9 (1): 1-82.

Ruta D, Garratt A \& Russell I (1999): Patient centred assessment of quality of life for patients with four common conditions. Quality in Health Care 8: 22-29.

Schipper H, Clinch J \& Olweny C (1996): Quality of life studies: Definitions and conceptual issues. Quality of Life and Pharmacoeconomics in Clinical Trials, $2^{\text {nd }}$ edition, pp. 11-23. Lippincott-Raven Publishers, Philadelphia.

Sneeuw K, Sprangers M \& Aaronson N (2002): The role of health care providers and significant others in evaluating the quality of life of patients with chronic disease. Journal of Clinical Epidemiology 55 (11): 11-25. 
Sparbel K \& Anderson M (2000a): Integrated literature review of continuity of care: Part 1, conceptual issues. Journal of Nursing Scholarship 32: 17-29.

Sparbel K \& Anderson M (2000b): Integrated literature review of continuity of care: Part 2, methodological issues. Journal of Nursing Scholarship 32: 131-137.

Speight J (2002): Assessing the impact of diabetes screening on quality of life or quality of health? Semantics are important. Diabetes Care 25(10): 1893-4.

Tengs T \& Lin T (2003): A meta-analysis of quality-of-life estimates for stroke. PharmacoEconomics 21(3): 191-200.

Theobald K \& McMurray A (2003): Successful recovery after CABG surgery: Lessons for discharge planning. Journal of Advanced Nursing (in press).

Ware J \& Sherbourne C (1992): The MOS-36 item Short-Form Health Survey (SF36) Medical Care 30: 473-83.

Wilkin D, Hallan L \& Doggett M (1992): Measures of Need and Outcome for Primary Health Care. Oxford Medical Publications, Oxford.

Williams B, Coyle J \& Healy D (1998): The meaning of patient satisfaction: An explanation of high reported levels. Social Science and Medicine 47 (9):13511359.

World Health Organization (2002): WHOQOL: Measuring Quality of Life, the World Health Organization Quality of Life Instruments (The WHOQOL-100 and the WHOQOL-BREF) http://www.org.int/msa/qol/ accessed April 4, 2003. 